\title{
REVISIÓN DE LA LITERATURA SOBRE DESEMPEÑO OCUPACIONAL DE TRABAJADORES EN PESCA COMERCIAL
}

\author{
REVIEW ABOUT OCCUPATIONAL PERFORMANCE \\ IN COMMERCIAL FISHING WORKERS
}

\section{Aránzazu Pacheco Martínez ${ }^{1}$ Ana Isabel Souto Gómez², Miguel Ángel Talavera Val- verde $^{3}$, Pedro Moruno Miralles ${ }^{4}$}

\begin{abstract}
Resumen:
Esta revisión tiene como propósito revelar los resultados de la búsqueda bibliográfica sobre desempeño ocupacional de trabajadores en pesca comercial. Su objetivo es realizar una aproximación sobre conceptos relacionados con el desempeño ocupacional de personas que dedican su actividad productiva a la pesca comercial de altura. Esta revisión bibliográfica se centra en personas que pertenecen a la comunidad autónoma de Galicia, situada al noroeste de España, la cual se caracteriza por guardar una fuerte vinculación con el sector de la pesca.

En cuanto a la metodología, se realizó una revisión bibliográfica en bases de datos Medline, CSIC, Biblioteca Virtual de Salud, World of Science (WOS), IBECS, Cochrane Plus, Econbiz, repositorios institucionales, monografías y literatura gris de alcance. La estrategia de búsqueda se ha basado en la utilización de descriptores controlados DECS y MESH. Los resultados reportados hacen referencia a las consecuencias derivadas del entorno en el desempeño ocupacional de los protagonistas. Cabe destacar la influencia de los aspectos tanto intrínsecos como extrínsecos del propio trabajo que desarrollan los trabajadores de la pesca, el cual repercute en su desempeño ocupacional. Como conclusiones, se han identificado modificaciones en la gestión de la ocupación produciendo una repercusión tanto en la persona como la estructura familiar; todo ello motivado por la actividad productiva desarrollada.
\end{abstract}

\section{Palabras clave:}

Terapia ocupacional, ocupación, desempeño ocupacional, salud, pesca comercial.

DECS: terapia ocupacional, ocupación, desempeño ocupacional, salud, pesca comercial.

\begin{abstract}
:
The purpose of this review is to show de results of the literature search about occupational performance of commercial fishing workers. The aim is to approach on concepts related to occupational performance of people who devote their productive activity to commercial fishing. This literature review focuses on Galician people, a community situated in the northwest of Spain, which
\end{abstract}

1 Graduada en Terapia Ocupacional. Dirección Postal: C/Baiona, nº3, 2izquierda. 36780. La Guardia, Pontevedra. Teléfono 667700643 e_mail:zazu33389@hotmail.com

2 Graduada en Terapia Ocupacional y Diplomada en Trabajo Social. Dirección Postal: c/Ricardo Santos. Edificio La Condesa. Portal 1. Número 7. Sanxenxo (Pontevedra) C.P. 36960.e_mail:anasogo@hotmail.es

3 Terapeuta Ocupacional. Xerencia de Xestión Integrada de Ferrol. Servicio Gallego de Salud. Profesor Asociado Universidad de la Coruña. Facultad Ciencias de la Salud. Director de Revista TOG www.revistatog.com

Dirección postal: Hospital Básico de Defensa. Hospital Naval. Unidad de Hospitalización Breve de Psiquiatría. Terapia Ocupacional. Crta. San Pedro de Leixa S/n. 15405. e_mail:miguelrevistatog@yahoo.es

4 Terapeuta Ocupacional. Doctor en Psicología. Profesor Titular de la Escuela Universitaria de la Facultad de Terapia ocupacional, Logopedia y Enfermería de Talavera de la Reina. Universidad de Castilla-La Mancha.Dirección postal:Avda. Real Fábrica de Seda, s/n 45600 Talavera de la Reina .Toledo e_mail:pedro.moruno@uclm.es 
is characterized by a strong link with fishing sector. In terms of methodology, the database used were: Medline , CSIC, Biblioteca Virtual de Salud, World of Science (WOS), IBECS, Cochrane Plus, Econbiz, institutional repositories, monographs and gray literature available. The search strategy was based on the use of controlled DECS and MESH descriptors. The results reported refer to the consequences of environment in occupational performance.

It has to be emphasized thatthe influence of both intrinsic and extrinsic aspects of work developed by fishingworkers, which affects their occupational performance. In conclusion, we have identified modifications in the management of the occupation producing an impact on both the individual and the family structure; all driven by the productive activity carried on by the protagonist.

\section{Keywords:}

Occupational therapy, occupation, occupational perfomance, health, comercial fishing.

MESH: occupational therapy, occupation, occupational perfomance, health, comercial fishing.

\section{INTRODUCCIÓN}

La ocupación, entendida como aquella actividad con sentido en la que la persona participa cotidianamente y que puede ser nombrada por la cultura es lo que da sentido a nuestra vida. Si consideramos que la ocupación, entre otras cosas, estructura nuestro quehacer, nuestra condición y nuestros tiempos, cuando alguno de estos factores no está presente, el desempeño ocupacional se resiente y éste genera en la persona una ausencia de la condición de salud.

El desempeño ocupacional es un motivo de estudio para los terapeutas ocupacionales y en la comunidad donde se encuentra enmarcado este estudio, éste tiene unas características muy concretas con respecto a las personas que trabajan en el mar.Galicia es una comunidad autónoma española situada en el noroeste de dicho país y rodeada de mar en tres de sus cuatro provincias, por lo que mantiene una fuerte vinculación con la pesca en muchas de sus modalidades: extracción, conservación o comercialización. Este hecho ha suscitado que desde las épocas más antiguas hasta la actualidad, Galicia represente un referente en el sector de la pesca, ya que sus 123 puertos la convierten en la pesquería más importante de España y de Europa (Losada. 2006). Además, la flota pesquera de Vigo se conforma como la más importante de Europa en capturas para el consumo humano, con un total de 742.204 toneladas de pescado (FUNDAMAR, 2006). De esta mane$\mathrm{ra}$, Vigo contribuye de manera fundamental a que Galicia sea la primera potencia pesquera de Euro- pa. Por todo ello, la pesca es una señal de identidad de esta ciudad.

Además, el puerto de Vigo supone para la ciudad el escenario de la mitad de la actividad económica (ARVI, 2013). El sector de la pesca extractiva tiene una gran importancia socioeconómica en esta ciudad debido a que emplea de manera directa a un total de 8.957 personas (ARVI, 2013) y teniendo en cuenta los empleos indirectos la población dependiente de este sector asciende a 32.679 personas (ARVI, 2013). Estas cifras, lo sitúan como el segundo sector productivo de Vigo, después de la automoción.

Todo ello, representa el $40 \%$ del Producto Interior Bruto (a partir de ahora será nombrado bajo las siglas PIB) (ARVI, 2013).

Por esta indiscutible transcendencia tanto a nivel local como autonómico, son muchos los actores influyentes sobre el trabajo en el mar; aspectos como los intereses económicos, la legislación, o las presiones medioambientales pueden influir en las características del trabajo de la pesca.

El desempeño ocupacional es definido por la Asociación Americana de Terapia Ocupacional (AOTA, 2008)como la capacidad de hacer y completar una actividad o una ocupación seleccionada como resultado de una transacción dinámica entre la persona, el contexto/entorno, y la actividad.

Un análisis del mismo, permite conocer si las áreas, los patrones de desempeño y los contextos o entornos pueden o podrían sufrir alguna modificación debido a factores extrínsecos o intrínsecos de la propia ocupación. 
Por tanto, podremos definir ocupación, como el conjunto de actividades personales, culturalmente significativas en las que los seres humanos se implican, nombradas en el léxico de la cultura (Moruno, Talavera, 2011). Además, es un estado inherente a la condición humana, dota de sentido a nuestra existencia, y está a su vez íntimamente relacionada con la salud, el bienestar y la calidad de vida (Moruno, Talavera 2011).

Son habituales las situaciones en las que se tiene que hacer frente a diversos cambios, éstas tienden a modificar el desempeño ocupacional. En el caso concreto de las personas que trabajan en el mar, y debido a las características tanto del propio trabajo como del entorno, estos cambios se ven acentuados. La adaptaciónentendida como un ajuste o compensación ("respuesta interna a una desafío significativo en el desempeño de roles") (Gómez Lillo, 2006), se debe poner en marcha cada día ya que en la vida a bordo de una barco, suceden situaciones y condiciones que no dependen directamente de la voluntad de las personas. Barris, Kielhofner, Levine \& Neville (2004) señalaron que "cultura incluye los valores, normas, costumbres, creencias, comportamientos y percepciones que son compartidas por un grupo o sociedad, bajo esta afirmación, una misma actividad puede tener un significado "culturalmente" distinto dependiendo de la cultura en la que tenga lugar, se podría hacer una comparación con los diferentes significados que se le atribuyen a actividades que se realizan en mar y en tierra.

En este caso, uno de los principales factores que influyen en el proceso de adaptación es el entorno social. La ocupación, no puede entenderse sin un entorno y un contexto, ni aislados de ellos. Concretamente en esta revisión, el contexto y el entorno, son condicionantes de los restantes componentes del desempeño ocupacional, en tanto que la ocupación de los implicados, depende lugar donde se desarrolla. Esto genera situaciones de riesgo para la salud, tales como la disminución de la fuerza, el aumento del tiempo de reacción, la coordinación, la dificultad en tomar decisiones, la pérdida de control sobre el equilibrio, alteraciones del estado de ánimo, problemas de a salud mental, aumento de enfermedades crónicas o la muerte prematura, entre otras. Todo ello, tiene lugar en un entorno y contextos cambiante, siendo esto una característica principal de este tipo de trabajo y generando una modificación y compromiso del desempeño ocupacional.

Considerando que la ocupación, estructura el quehacer y los tiempos genera salud, cuando alguno de los factores que componen el desempeño ocupacional no está presente o se ve modificado, como es este caso donde la situación repercute en la persona de forma directa sobre su condición de salud.

¿Qué entendemos por condición de salud? La salud abordada desde un enfoque biopsicosocial, proporciona una visión desde una perspectiva tanto biológica, individual y social, teniendo en cuenta los factores ambientales que pueden ejercer un efecto en todos los componentes del funcionamiento. Además, la condición de salud es un recurso para la vida diaria, para desempeñar la ocupación de una manera satisfactoria y disfrutar de ella, brindándonos diferentes oportunidades. Es por tanto un estado de bienestar físico, mental y social, no solo ausencia de enfermedad.

Complementando estos conceptos de salud, Wilcock (1998) añade que "las actividades (...) no necesariamente son positivas para la salud y el bienestar de los sujetos", y esto guarda relación con las condiciones en las que tiene lugar la ocupación de los trabajadores del mar, ya que debido a estas características mencionadas se pueden dar repercusiones tanto en la salud como en el bienestar. Además, no podemos perder de vista, que en muchas definiciones de ocupación se destaca ésta como agente de salud y bienestar incluso, Wilcock (1998) afirma que la salud, el bienestar y la satisfacción ocupacional son más importantes que el crecimiento económico.

El mar es un entorno hostil en el cual con mucha frecuencia tienen lugar accidentes peligrosos, y debido a las circunstancias en las que suceden, el acceso a los servicios de salud se suele hacer 
dificultosa ya que no existe cobertura sanitaria a buques en todos los océanos del mundo.

Así, las condiciones de trabajo en alta mar, llevadas a término en circunstancias climáticas límites, reducida habitabilidad, aislamiento, reducción de la interacción personal, problemas de alumbrado o ventilación, golpes contra objetos en movimiento, ruido de la sala de máquinas o caídas al mar no son las más favorables para mantener una condición óptima de salud. Trabajar en alta mar, presenta unas condiciones determinadas que hacen que esta actividad se convierta en una de las más peligrosas y de las que mayor repercusión generan en la salud de los que la practican (FUNDAMAR, 2004). El sector de la pesca, engloba un conjunto de actividades muy amplio y diferente, con múltiples subsectores, artes, flotas, entre otros. Dentro de las distintas ramas que comprende el sector pesquero, esta revisión se centra en la pesca extractiva; una actividad donde la relación de los humanos con el recurso, se limita a su localización y captura.

El principal escenario de observación de esta revisión es la presencia de un buque como centro, tanto de trabajo como de vivienda. En cuanto al trabajo, la pesca supone la realización, durante largas horas, de tareas extenuantes en un medio hostil, como es el mar, a ello se le suma el alejamiento del trabajador de su domicilio y de sus familias durante largos períodos de tiempo. Además, cabe destacar las peculiaridades de las condiciones sociales y laborales que sitúan a este colectivo entre los que sufren un mayor deterioro físico y psíquico, envejeciendo con mayor rapidez (FUNDAMAR, 2004).

El cuanto al buque como vivienda, debido a cuestiones de productividad en el aprovechamiento de espacio, abundan las zonas reducidas e incómodas, ya que los diseños de habitabilidad suelen ser bastante pobres en beneficio de los de almacenaje. El buque de pesca, a diferencia de otros como los de la marina militar, se sitúa, en el extremo final del diseño de la habitabilidad. Estos aspectos estructurales, condicionan a otros como son la falta de intimidad o de espacio individual, que unidos a la sobrecarga de trabajo y la larga permanencia en la mar, generan un fuerte desarraigo social en los trabajadores. La revisión concluye que esta situación empeora si la tripulación es heterogénea, es decir, formada por más de un grupo étnico; en cuyo caso la poca comunicación se limita a la actividad extractiva, impidiendo o dificultando la formación de un grupo y una adecuada convivencia.

Además de las características más adversas mencionadas hasta ahora, el trabajo en alta mar posee una condición muy exclusiva que lo diferencia de otros trabajos y es que se encuentra inmerso en una cultura, "la cultura del mar", que lo convierte en un modo de vida, y en un sistema de ideas y sentimientos. No se puede entender ocupación sin cultura: "la ocupación es un proceso dinámico, en la que interviene la cultura a laque pertenece una persona". Esa cultura le asigna significados a lo que la persona hace por tanto, la ocupación es una síntesis de ser, hacer y llegar a ser.

Esta revisión se centra en el análisis del desempeño ocupacional de las personas que trabajan en buques con actividad pesquera de altura. Además, se estudiará la relación del entorno con la salud. Por tanto, se realiza un análisis exhaustivo de la ocupación y su significado, partiendo del supuesto básico de que el ser humano es un ser ocupacional y que la ocupación, entre otras cosas, estructura nuestro quehacer, nuestra condición y nuestros tiempos, siendo el eje central de nuestra vida.

\section{MetodoloGía}

En el diseño de este estudio se ha realizado una revisión de documentos, libros y artículos a través de una búsqueda bibliográfica la cual se ha centrado tanto en bases de datos relacionadas con las ciencias de la salud como en otras de disciplinas fines: IBECS, CSIC, PUBMED, WORLD OF KNOWLEDGE, CROCHANE, ECONBIZ y INSHT. Además, se han consultado repositorios institucionales, monografías y literatura gris de alcance. 
La estrategia de búsqueda se ha basado en la utilización términos libres: pesca, pesca de altura, pesca comercial, empleo, significado, trabajadores del mar y de DECS y MEHS siendo los primeros: "ocupación, terapia ocupacional" y "salud" y los términos MESH "occupation", "occupational therapy" y "health", todo ello con el objetivo ampliar la localización de documentación. Esta búsqueda se ha realizado tanto en español como en ingles. Además, fueron analizadas las referencias bibliográficas de los artículos seleccionados con el fin de rescatar otros estudios potencialmente incluibles para la revisión.Se utilizó para la redacción de la estrategia de búsqueda los operadores booleanos "AND" y "OR". La duración de la búsqueda bibliográfica fue de 3 meses, desde octubre a diciembre.

Una vez finalizado este proceso, se establecieron los siguientes criterios de inclusión y exclusión, con el fin de agilizar la lectura y concretar los más relevantes:

- Criterios de inclusión:

- Artículos científicos en los que se describe el desempeño ocupacional en la pesca comercial, apareciendo este tipo de descripción en su resumen.

- Artículos dónde se recoja mediante metodología cualitativa y cuantitativa, experiencias de vida, testimonios o evidencias de los efectos del desempleo en las personas que realizan una actividad de pesca comercial, apareciendo este tipo de descripción en su resumen.

- Se ha aplicado una limitación temporal de tal manera que únicamente se han utilizado artículos publicados entre 2003 y 2013.

- Artículos en idioma inglés y castellano.

- Criterios de exclusión:

- Se han excluido artículos de opinión, cartas al director y editoriales.

- Artículos con una antigüedad mayor a 10 años.

- Artículos escritos en un idioma que no fuera español o inglés.

Tras la búsqueda inicial se localizaron 75 estudios, aunque se excluyeron 23 que no fueron relevantes para el objetivo de esta revisióndebido a que su contenido no se ajustaba a los propósitos de esta revisión. Por tanto, el número final seleccionado fue de 52 Artículos.

\section{RESULTADOS}

Los principales hallazgos obtenidos tras la realización de la revisión de la literatura muestran como las características del trabajo en el mar, tales como el entorno en el que tiene lugar, las condiciones del propio trabajo, o el aislamiento, pueden tener repercusiones en la salud de los trabajadores, pudiendo provocar una modificación en el estado de la misma.

Los aspectos más relevantes, se pueden resumir en el buque como centro de trabajo y vivienda, la consecuente lejanía del hogar, el mar como entorno principal de desempeño ocupacional, las condiciones climatológicas en las que desarrollan la ocupación y las tareas de pesca como eje en torno al cual gira y se desarrolla el día a día(Carril, 2006).

El contexto y el entorno en el que este trabajo tiene lugar cuenta con unas características específicas que lo diferencias de la mayoría de los trabajos; estos son: las circunstancias climáticas límites, la reducida habitabilidad, el aislamiento, la reducción de la interacción personal, problemas de alumbrado o ventilación, los golpes contra objetos en movimiento, el ruido de la sala de máquinas o las caídas al mar entre otros (Hervás Rivero et al. 2014).

En este entorno (que cuenta con peculiaridades tales como medio físico en movimiento, espacios reducidos, jornadas prolongadas y ritmo de trabajo intenso y elevado, entre otros)y haciendo referencia a los resultados obtenidos ocurren todos los aspectos de la ocupación, es decir el hombre "vive, trabaja y muere en su interior", pero también las convierte en lugar de ocio y recreo, aunque en esta revisión los informantes no hacen especial hincapié en este área convirtiéndolo en un tiempo poco satisfactorio, si lo comparan con 
el tiempo que pasan en tierra. El entorno físico en un buque se encuentra condicionado por aspectos de productividad y rendimiento, no por su habitabilidad. Esto tiene unas repercusiones directas en la forma de mantener el desempeño ocupacional ya que los espacios son muy limitados y existe una exposición continua a factores de riesgo potenciales. Además, la persona no es protagonista ni tiene autonomía a la hora de adaptar el entorno a sus condiciones, deseos y voluntades. Es por esto, que el entorno hostil en el que se desarrolla la ocupación de los protagonistas (debido principalmente a las limitaciones del entorno y a los espacios restringidos), genera consecuencias en la competencia y equilibrio ocupacional teniendo repercusiones en su desempeño ocupacional (San Valer, Rubio Ortega, 2013).

Además, el cambio de entorno que vive una persona que ha estado embarcada durante un periodo de tiempo cuando vuelve a su hogar, provoca una repercusión directa en: áreas de ocupación, como consecuencia de una nueva adaptación a los patrones de desempeño para desempeñar la ocupación en los contextos y entornos en tierra, en los que la ocupación tiene lugar.

Por este motivo, la persona embarcada deja de ejercer roles, presentando una modificación del desempeño de los mismos, en un entorno que a la vuelta de este protagonista, mantuvo su gestión de forma independiente al margen de esta persona. El trabajo en alta mar tiene repercusiones en la ocupación de las personas que lo realizan, así como en la de su familia, ya que éstas sufren a su vez, una pérdida temporal de uno de sus miembros, con la consecuente pérdida de roles. Por lo que una modificación en ellos, puede suponer tanto para la persona como para su entorno más cercano, una disrupción de los comportamientos que se espera de ellos, generando repercusiones en el devenir del entorno personal, entre otros (Carril, 2006).

Núñez (2012) explica como todo lo que hacen los seres humanos existe dentro del tiempo, ya que éste es percibido como un vacío que se nos hace necesario llenar con las cosas que ha- cemos, de forma que nuestro hacer se despliega en el tiempo y va llenando el presente. De hecho, muchas veces sabemos lo que tenemos que hacer en base a la medición del tiempo, en el caso de las rutinas diarias. Los principales resultados obtenidos en cuanto a patrones de desempeño tales como hábitos, rutinas o roles, ponen de manifiesto que éste se ve reducido a la mínima expresión a bordo de un buque. La rutina y la monotonía provocan una pérdida de consciencia de aspectos temporales como los días de la semana. Además, debido a esta pérdida, son otros los aspectos que marcan el tiempo en un buque como pueden ser el tipo de comida (en la mayoría de los buques gallegos es tradición que los domingo haya cocido para comer) o el periodo lunar.

Estudios realizados en ciudades costeras de América del Norte (Nguyen y Leung, 2006; (Lawrie, Matheson, Murphy, Ritchie, Bond, 2003) definen el entorno del trabajo del mar como un lugar hostil donde se pueden ver obstaculizadas actividades, (descanso o sueño) a la vez que se amplían las dificultades para el desempeño de otras (actividad laboral o participación social). Además en cuanto a las limitaciones que subyacen de un entorno como el de un buque en alta mar, son los espacios y las relaciones sociales. Las relaciones que tienen lugar en estas condiciones, son siempre las mismas, no varían, llegando a generar conflictos y problemas en la gestión de las interacciones, repercutiendo en la fluidez de la participación social.

El cuanto al buque como vivienda, debido a cuestiones de productividad en el aprovechamiento de espacio, abundan las zonas reducidas e incómodas, ya que los diseños de habitabilidad suelen ser bastante pobres en beneficio de los de almacenaje. El buque de pesca, a diferencia de otros como los de la marina militar, se sitúa, en el extremo final del diseño de la habitabilidad. Estos aspectos estructurales, condicionan a otros como son la falta de intimidad o de espacio individual, que unidos a la sobrecarga de trabajo y la larga permanencia en la mar, generan un fuerte desarraigo social en los trabajadores. 
Pueden ser múltiples causas las que influyen en esta situación, pero en este caso, entre otras cosas, es debido a: las condiciones que experimentan estando embarcados y los diferentes factores que influyen en el desarrollo del trabajo. De la misma manera, los protagonistas viven de manera muy diferente su desempeño ocupacional estando a bordo y en tierra. Los resultados muestran como muchas de las actividades que se realizan a bordo, están influidas por diferentes aspectos implícitos del trabajo en un buque de altura. Los aspectos más relevantes, se pueden resumir en el buque como centro de trabajo y vivienda, la consecuente lejanía del hogar, el mar como entorno1 principal de desempeño ocupacional, las condiciones climatológicas en las que desarrollan la ocupación de los protagonistas y las tareas de pesca como eje en torno al cual gira y se desarrolla el día a día.

\section{DISCUSIÓN}

La revisión de la literatura realizada demuestra que el trabajo en el mar debido a sus características podría tener efectos en la gestión de la ocupación motivados por los constantes cambios de entorno y provocando una posible alteración en la salud. Además, aspectos de la ocupación como las áreas, los patrones de desempeño o los contextos y entornos pueden o podrían sufrir alguna alteración debido a factores extrínsecos e intrínsecos de la propia ocupación.

Cabe destacar el vacío científico con respecto al desempeño ocupacional de personas que trabajan en buques con actividad pesquera de altura. En general, es escasa la literatura que relacione el trabajo en el mar y los aspectos más socio-sanitarios de éste, y sin embargo esta evidencia es más notable en estudios relacionados con la marina mercante. Este tipo de pesca cuenta con una normativa más estricta y desarrollada que la pesca comercial y esto ha provocado que los estudios en este ámbito sean más prevalentes.

Los resultados reportados, añaden que en este entorno (que cuenta con peculiaridades tales como medio físico en movimiento, espacios reducidos, jornadas prolongadas y ritmo de trabajo intenso y elevado, entre otros) y haciendo referencia a los resultados obtenidos ocurren todos los aspectos de la ocupación, es decir el hombre "vive, trabaja y muere en su interior", pero también las convierte en lugar de ocio y recreo.

El entornoy sus características, son los responsables de que exista una baja competencia ocupacional en cuanto a su participación en actividades o formas ocupacionales (Navarrete, 2013). Esto, coincide con lo aportado por Wilcock (2006), la cual asevera que la deprivación ocupacional tiene lugar cuando existen limitaciones o circunstancias que no permiten que la persona adquiera, utilice o disfrute de la actividad, apareciendo un nivel de alienación ocupacional que produce una falta de satisfacción personal, a pesar de haber participación en la ocupación.

El entorno físico en un buque se encuentra condicionado por aspectos de productividad y rendimiento, no por su habitabilidad. Esto tiene unas repercusiones directas en la forma de mantener el desempeño ocupacional1 ya que los espacios son muy limitados y existe una exposición continua a factores de riesgo potenciales.

En términos generales, los cambios que sufre una persona embarcada cuando retorna a su hogar durante periodos más o menos largos, van a requerir un consecuente periodo de adaptación a ellos, y son variadas las causas de los mismos. Navarrete (2013), defiende que de la mano de los cambios va siempre la necesidad de adaptación. Gómez Lillo (2006), entiende adaptación como una respuesta interna a un desafío significativo en el desempeño de roles. En este sentido, un ejemplo lo constituiría la modificación en el cumplimiento de roles en tierra La vida de una persona que se dedica a la pesca está llena de cambios, se producen cambios de actividades, de patrones de desempeño, de todas las áreas de la ocupación y sobre todo de cómo perciben el significado de los mismos. Los resultados obtenidos reflejan que existe un conformismo y una adaptación que se ha ido estructurando a lo largo de los años. Del 
mismo modo lo corrobora un estudio realizado en pescadores vietnamitas, el cual concluye que los pescadores se han adaptado a su entorno único. Según Navarrete (2013), la necesidad de adaptación está íntimamente ligada a la capacidad de estructurar y gestionar la ocupación y esto facilita a su vez, la capacidad de dar forma a la vida de las personas. En relación a ello, ante el planteamiento sobre del interés de conseguir un empleo en tierra, la mayoría de los testimonios tienen como factor común lo que les costaría adaptarse a los patrones de desempeño ocupacional. De la misma manera, se justifican hablando sobre las vacaciones y la libertad que les ofrece la vida del mar.

El mar es un entorno hostil en el cual con mucha frecuencia tienen lugar accidentes peligrosos, y debido a las circunstancias en las que suceden, el acceso a los servicios de salud se suele hacer dificultosa ya que no existe cobertura sanitaria a buques en todos los océanos del mundo. Así, las condiciones de trabajo en alta mar, llevadas a término en circunstancias climáticas límites, reducida habitabilidad, aislamiento, reducción de la interacción personal, problemas de alumbrado o ventilación, golpes contra objetos en movimiento, ruido de la sala de máquinas o caídas al mar no son las más favorables para mantener una condición optima de salud (OMS, 2001).

Estar en alta mar durante largos periodos para que la persona se desvincule físicamente de su familia y de su casa. Todo ello, unido a unas características del entorno hostil donde se desempeña su actividad laboral, cambiante al Ilegar a tierra, puede desembocar en una situación de deprivación ocupacional como consecuencia de una restricción en la opción de tener oportunidades en cuanto a la gestión de la ocupación. Por tanto, en este caso, podemos narrar que el cambio de entorno y sus características sería el factor que condicionaría el estado de deprivación ocupacional (Sanz y Rubio, 2011).

El otro concepto que cabe matizar en la discusión de resultados es el de cultura. Por un lado se encuentra una clara influencia del contexto cultural en cuanto a la forma de desarrollar los patrones de desempeño o la participación social, aunque de todos ellos cabe destacar el fuerte impacto en la convivencia. Navarrete (2013) citando a Wilcock afirma que la cultura influye en la ocupación de las personas, ya que ésta le asigna significado a lo que la persona hace. En este caso, muchos informantes hacían referencia al cambio de actividades que desarrollaban, debido a la incorporación de tripulaciones extranjeras a los buques.

Según Suárez de Vivero (2003), en pesqueros, el factor convivencia es muy severo dado la falta de espacio individual, la sobrecarga de trabajo y la larga permanencia en la mar que genera un fuerte desarraigo social en los tripulantes de este tipo de buques. La situación vivencial se empeora si la tripulación es mixta, es decir, la formada por más de un grupo étnico; en este caso la incomunicación es grave y la realidad cotidiana de dos o más grupos con un solo nexo de unión, la actividad extractiva, no facilita la formación de un sustrato favorable a una adecuada cohabitación.

Por otro lado, el trabajo de la pesca de altura ha sufrido grandes avances y mejoras en el periodo de los últimos 20 a 30 años y aunque la aparición de las nuevas tecnologías a bordo, ha generado una retracción de la socialización, la participación y las relaciones, el avance a resultado prometedor y meteórico en legislación. En primer lugar, en la actualidad la legislación, que es más sólida en materia de derechos fundamentales a bordo y calidad de vida. En segundo lugar, el devenir de las mejoras en el transcurso de los años dedicados a esta actividad, ha generado un ajuste interno en cada empresa en estos aspectos, primando y dando valor a las comodidades de los trabajadores (INSHT, 2014).

Un ejemplo de ello lo constituye la incorporación de potabilizadoras a bordo. Este avance ha propiciado un cambio en las actividades de aseo, como consecuencia de un cambio de patrones de desempeño relacionado con el proceso de transformación e innovación de los buques, en tanto que en la actualidad, todos los buques Ilevan potabilizadoras de agua, las cuales permiten un uso ilimitado de la misma. Por tanto, una actividad 
que antaño era una excepción, se ha convertido en algo rutinario y normalizado que se ha automatizado, generando un hábito.

Además, estos avances se han consolidado también a nivel internacional fomentado por el esfuerzo realizado desde la Organización Internacional del Trabajo(OIT) y desde la Organización Marítima Internacional(OMI), quienes alentaron a que el Parlamento Europeo desarrollase (en el año 2014) normativa y legislación de Política Pesquera Común (PPC) donde por primera vez se han tenido en cuenta al mismo nivel que los económicos, los aspectos sociales y los medioambientales (OMI, 2014).

Por otro lado, existen otros factores que podrían provocar cambios de la condición de salud, incluyendo aspectos tales como: lesiones por cargas físicas importantes, ausencia de descanso regulado, turnos de trabajo largos e irregulares, cambios en la alimentación, exigencias elevadas, o accidentes de trabajo, entre otros. Todo ello aumenta el número, la posibilidad o la gravedad de las enfermedades y de los accidentes de origen laboral que sufren los trabajadores, desembocando en situaciones de estrés o fallecimientos, entre otros

Por todo ello, y para finalizar, podemos concluir que esta revisión muestra cómo, por un lado, el entorno donde se lleva a cabo una actividad productiva es influyente y determinante tanto para la persona como para su núcleo y estructura familiar; determinando tanto la calidad de las relaciones como la periodicidad de las mismas. Asimismo, el entorno produce cambios en el desempeño ocupacional (hábitos y rutinas) y en la gestión de la ocupación, conllevando a un desequilibrio ocupacional que repercute en la salud de las personas que ejercen un trabajo en alta mar. Estos cambios repercuten y activan un proceso de adaptación tanto en las protagonistas como en las personas de su entorno.

Por último, cabe destacar que la investigación de terapia ocupacional y otras disciplinas afines con respecto a este tema son muy limitadas, habiendo un especial vacío de estudios relacionados con el desempeño ocupacional de las personas embarcadas, y la relación entre la salud, seguridad y condiciones de trabajo, los cuales si fueran conocidos podrían ser abordados, evitando así que puedan presentar a lo largo de su vida alguna dificultad en el desempeño ocupacional y por consiguiente en la salud.

\section{REFERENCIAS BIBLIOGRÁFICAS}

Álvarez, E., Gómez, S., Muñoz, I., Navarrete, E., Riveros, M.E., Rueda, L., et al. (2007). Definición y desarrollo del concepto de ocupación: ensayo sobre la experiencia de construcción teórica desde una identidad local. Revista Chilena de Terapia Ocupacional.7: 76-82.

American Occupational Therapy Association. (2008). Occupational therapy practice framework: Domain and process (2nd ed.). American Journal of Occupational Therapy.62: 625-83.

Bond, C., Lawrie, T.,Matheson, C., Murphy, E., Ritchie, L. (2004). The health and lifestyle of Scottish fishermen: a need for health promotion. Health Education Research.19(4): 373-79.

Carril Vázquez, X.M. (2006). Algunas de las especificidades más problemáticas del trabajo en el mar realizado a bordo de embarcaciones pesqueras. Revista Galegade Economía (Galicia). 15(1): $1-15$.

Gómez Lillo, S. (2006). Equilibrio y organización de la rutina diaria. Revista Chilena de Terapia Ocupacional.6: 47-54.

Hervás Rivero, P., Díaz Aramburu, C., De Vicente Abad, A., De la Orden Rivera, MV. Moreno Reyes, F.J., Muñoz Nieto-Sandoval, M., et al. (2014). Sector pesquero: análisis de situación de la salud laboral. Madrid: Instituto Nacional de Seguridad e Higiene en el Trabajo (INSHT).

Jerry Dzugan, M.E. (2010). The Development and Efficacy of Safety Training for Commercial Fishermen. Journal of agromedicine.15(4):1-6.

Lincoln, J.M., Conway, G.A. (1999). Preventing commercial fishing deaths in Alaska. Occupational Enviroment Medical.56: 691-95.

Lincoln, J.M., Lucas, D.L. (2010). Fatal Occupational Injuries in the U.S. Commercial Fishing Industry: Risk Factors and Recommendations. Anchorage: National Institute for Occupational Safety and Health. Publication Number: 2011-103.

Lincoln, J.M., Lucas, D.L. (2010). Occupational fatalities in the United States commercial fishing industry, 2000-2009. MMWR. Morbidity and mortality weekly report.59(27): 842-45

Losada Soutelo, C.M. (2006). El papel de los puertos en el desarrollo del sector pesquero: el puerto de Vigo. Revista Galega de Economía (Galicia).15(1): 1-8.

Lucas, D.L., Lincoln, J.M. (2007). Fatal falls overboard on commercial fishing vessels in Alaska. American journal of industrial medicine.50(12):962-968.

Moruno Miralles, P. Talavera Valverde, M.A. (2011). Ocupación y calidad de vida. En: Moruno Miralles P, Talavera Valverde MA, 
coordinadores. Terapia Ocupacional en Salud Mental, (pp. 2136). Barcelona: Elsevier Masson.

Moruno Miralles, P., Fernández Agudo, P. (2012). Análisis teórico de los conceptos privación, alienación y justicia ocupacional. TOG (A Coruña), 9(monog. 5): 44-68. Recuperado el25-10-2013 en: http://www.revistatog.com/mono/num5/teorico.pdf

Navarrete Salas, E. (2010). Ciencia de la Ocupación: caminos y perspectivas. TOG (A Coruña), 7(Supl. 6) : p 132-43. Recuperado el 3-12-2013 en: http://www.revistatog.com/suple/num6/erna.pdf

Nguyen, Q., Leung, P. (2013). Do Fishermen Have Different Attitudes Toward Risk? An Application of Prospect. Theory to the Study of Vietnamese Fishermen. The Australian journal of agricultural and resource economics. 34(3):518-538.

Núñez Paz, A. (2012). Ocupación, cultura y sociedad: el entorno social y cultural como clave para el éxito de la Terapia Ocupacional. TOG (A Coruña),9(monog. 5): 32-43. Recuperado el 17-102013 en:http://www.revistatog.com/mono/num5/clave.pdf

Organización Marítima Internacional. (2001). Orientaciones acerca de la reducción y gestión de la fatiga. Londres: OMI. MSC/ Circ.1014.

Organización Mundial de la Salud. (2001). Clasificación Internacional del Funcionamiento, de la discapacidad y de la Salud: CIF. Madrid: Ministerio de Trabajo y Asuntos Sociales.
Poggie, J.J., Pollnac, R.B. (2008). Happiness, well-being and psychocultural adaptation to the stresses associated with marine fishing. Human ecology review. 15(2):194-200

Pollnac, R., Bavinck, M., Monnereau, I. (2012). Job Satisfaction in Fisheries Compared. Social indicators research. 109:119-133.

Rodríguez Rodríguez, J.A., Suárez de Vivero, J.L. (2003). El buque como vivienda: Una aproximación metodológica. Scripta Nova, Revista Electrónica de Geografía y Ciencias Sociales.7(149): [28p.]. Recuperado el 6-11-2013 en: http://www.ub.es/geocrit/sn/sn-149.htm

Sanz Valer, P., Rubio Ortega, C. (2011). Ann Allart Wilcock: trayectoria profesional y aportaciones más relevantes a la terapia ocupacional y a la ciencia de la ocupación. TOG (A Coruña),8(14): 1-18. Recuperado el 5-09-2013 en: http://www.revistatog.com/ num14/pdfs/historia1.pdf

Valderrama Núñez, C., Lara Riquelme, P. (2013). Cuestionamientos sobre el carácter beneficioso para la salud y el bienestar de la ocupación: la emergencia de la ocupación como fenómeno social. TOG (A Coruña), 10(18): 1-15. Recuperado el 3-10-2013 en:http://www.revistatog.com/num18/pdfs/original9.pdf

Whalley Hammell, K.R., Iwama, M. (2012). Well-being and occupational rights: An imperative for critical occupational therapy. Scandinavian journal of occupational therapy. 19: 385-394

Wilcock, A.A. (1998). An occupational perspective of health. Thorofare, NJ: Slack. 